NUMBER THEORY WEEK 2017

BANACH CENTER PUBLICATIONS, VOLUME 118

INSTITUTE OF MATHEMATICS

POLISH ACADEMY OF SCIENCES

WARSZAWA 2019

\title{
ON THE RANKIN-SELBERG CONVOLUTION OF DEGREE 2 FUNCTIONS FROM THE EXTENDED SELBERG CLASS
}

\author{
JERZY KACZOROWSKI \\ Faculty of Mathematics and Computer Science, Adam Mickiewicz University \\ Umultowska 87, 61-614 Poznań, Poland; \\ Institute of Mathematics of the Polish Academy of Sciences \\ Sniadeckich 8, 00-656 Warsaw, Poland \\ E-mail: kjerzy@amu.edu.pl \\ ALBERTO PERELLI \\ Dipartimento di Matematica, Università di Genova \\ via Dodecaneso 35, 16146 Genova, Italy \\ E-mail: perelli@dima.unige.it
}

\begin{abstract}
Let $F(s)$ be a function of degree 2 from the extended Selberg class. Assuming certain bounds for the shifted convolution sums associated with $F(s)$, we prove that the Rankin-Selberg convolution $F \otimes \bar{F}(s)$ has holomorphic continuation to the half-plane $\sigma>\theta$ apart from a simple pole at $s=1$, where $1 / 2<\theta<1$ depends on the above mentioned bounds.
\end{abstract}

1. Introduction. It is well known that given two normalized Hecke eigenforms $f, g$ of weight $k$ and level 1, with Fourier coefficients $a(n)$ and $b(n)$, respectively, the associated Rankin-Selberg convolution

$$
L(s, f \otimes \bar{g})=\sum_{n=1}^{\infty} \frac{a(n) \overline{b(n)}}{n^{s}}
$$

has meromorphic continuation to the whole complex plane. Moreover, $L(s, f \otimes \bar{g})$ has a simple pole at $s=1$ if and only if $f=g$; see Chapter 13 of Iwaniec [2]. It is also well

2010 Mathematics Subject Classification: Primary 11M41, 11F30.

Key words and phrases: Selberg class, Rankin-Selberg convolution, shifted convolution sums. The paper is in final form and no version of it will be published elsewhere. 
known that the Rankin-Selberg convolution has been generalized and extended in several important directions.

Given a function $F(s)$ from the extended Selberg class $\mathcal{S}^{\sharp}$ with Dirichlet coefficients $a(n)$, see below for definitions, its Rankin-Selberg convolution is defined as

$$
F \otimes \bar{F}(s)=\sum_{n=1}^{\infty} \frac{|a(n)|^{2}}{n^{s}} .
$$

Very little is known about $F \otimes \bar{F}(s)$ in the general framework of $\mathcal{S}^{\sharp}$. Actually, as far as we know, the only result of general nature on $F \otimes \bar{F}(s)$ appears in Lemma $\mathrm{C}$ of [6], where we proved that if $F(s)$ has degree $1<d_{F}<2$, then $F \otimes \bar{F}(s)$ is holomorphic for $\sigma>1-\delta$ apart from a simple pole at $s=1$, where $\delta$ is a certain positive constant. However, we have later shown in [8] that there exist no functions in $\mathcal{S}^{\sharp}$ with $1<d_{F}<2$, and apparently the techniques that we used in Lemma $\mathrm{C}$ are not applicable for similar investigations of functions with degree $d_{F} \geq 2$ without injecting new ideas. Hence the problem of the behavior of $F \otimes \bar{F}(s)$ for general functions $F(s)$ of degree $d_{F} \geq 2$ is widely open, although we may ask if any result can be obtained in the case $d_{F}=2$, which is on the border of the range we treated previously, by elaborating on the techniques in [6].

In this paper we prove a conditional result about the meromorphic continuation of $F \otimes \bar{F}(s)$ for all functions of degree 2 in $\mathcal{S}^{\sharp}$. Our assumption concerns suitable bounds for the shifted convolution sums

$$
S_{k}(x)=\sum_{n \leq x} a(n) \overline{a(n+k)}
$$

with integers $k \geq 1$. These sums are classical objects, and non-trivial information about their behavior as $x \rightarrow \infty$ is known in several concrete cases. We shall assume bounds of type

$$
S_{k}(x) \ll x^{\theta},
$$

see 1.3 below, while the Cauchy-Schwarz inequality immediately shows that, uniformly in $x$ and $k$,

$$
S_{k}(x) \ll x^{\left(\sigma_{a}(F \otimes \bar{F})+\varepsilon\right) / 2}(x+k)^{\left(\sigma_{a}(F \otimes \bar{F})+\varepsilon\right) / 2},
$$

where $\sigma_{a}(F \otimes \bar{F})$ is the abscissa of absolute convergence of $F \otimes \bar{F}(s)$ and $\varepsilon>0$ is arbitrarily small. Actually, in general this bound is essentially optimal, since for example in the case of the divisor function $d(n)$ we have, for fixed $k$ and $x \rightarrow \infty$,

$$
\sum_{n \leq x} d(n) d(n+k) \sim x P_{k}(\log x)
$$

with certain polynomials $P_{k}(X)$, and $\sigma_{a}(F \otimes \bar{F})=1$ in this case. However, in several concrete examples of degree $2 L$-functions, $S_{k}(x)$ has no main term and non-trivial bounds are known with $\theta<1$; see e.g. Corollary in Jutila [3]. Therefore, some links exist between the $S_{k}(x)$ 's and $F \otimes \bar{F}(s)$, but obtaining information on the analytic continuation of $F \otimes \bar{F}(s)$ to the left of $\sigma_{a}(F \otimes \bar{F})$ from suitable bounds for $S_{k}(x), k \geq 1$, is a non-trivial problem. Here we propose an approach to this question. 
The extended Selberg class $\mathcal{S}^{\sharp}$ consists of the Dirichlet series $F(s)$ absolutely convergent for $\sigma>1$ and such that $(s-1)^{m} F(s)$ extends to an entire function of finite order for some integer $m$, satisfying the functional equation $\Phi(s)=\omega \overline{\Phi(1-\bar{s})}$ with $|\omega|=1$ and

$$
\Phi(s)=Q^{s} \prod_{j=1}^{r} \Gamma\left(\lambda_{j} s+\mu_{j}\right) F(s),
$$

where $Q, \lambda_{j}>0$ and $\Re \mu_{j} \geq 0$. Degree, conductor and $\xi$-invariant of $F \in \mathcal{S}^{\sharp}$ are respectively defined as

$$
d_{F}=2 \sum_{j=1}^{r} \lambda_{j}, \quad q_{F}=(2 \pi)^{d_{F}} Q^{2} \prod_{j=1}^{r} \lambda_{j}^{2 \lambda_{j}}, \quad \xi_{F}=2 \sum_{j=1}^{r}\left(\mu_{j}-1 / 2\right)=\eta_{F}+i d_{F} \theta_{F},
$$

say; here we deal only with functions of degree $d_{F}=2$, and for simplicity we denote the conductor simply by $q$. We refer to our surveys [4, [5], [11, [12, [13] and [14] for definitions and the basic theory of the Selberg class. For $F \in \mathcal{S}^{\sharp}$ and an integer $k \geq 0$ we define

$$
F_{k}(s)=F \otimes_{k} \bar{F}(s)=\sum_{n=1}^{\infty} \frac{a(n) \overline{a(n+k)}}{n^{s}} ;
$$

clearly $F \otimes \bar{F}(s)=F_{0}(s)$, and from the Cauchy-Schwarz inequality we see that $F_{k}(s)$ is absolutely convergent for $\sigma>\sigma_{a}(F \otimes \bar{F})$.

As outlined before, we assume that as $x \rightarrow \infty$

$$
S_{k}(x) \ll_{\varepsilon, \theta} x^{\theta} \quad \text { uniformly for } 1 \leq k \leq x^{\varepsilon},
$$

where $\theta \in \mathbb{R}$ and $\varepsilon>0$ is arbitrarily small.

TheOREM. Let $F \in \mathcal{S}^{\sharp}$ with degree 2 satisfy $(1.3)$ with some $1 / 2<\theta<\sigma_{a}(F \otimes \bar{F})$. Then $\sigma_{a}(F \otimes \bar{F})=1$, and $F \otimes \bar{F}(s)$ has holomorphic continuation to $\sigma>\theta$ apart from a simple pole at $s=1$.

Recalling that the Ramanujan condition states that $a(n) \ll n^{\varepsilon}$ for every $\varepsilon>0$, we have the following corollary.

COROLlary. Under the same hypotheses, there exists a constant $c_{0}>0$ such that

$$
\sum_{n \leq x}|a(n)|^{2} \sim c_{0} x
$$

Moreover, if $F(s)$ satisfies the Ramanujan condition, then $\sigma_{a}(F)=1$.

REMARKS.

1. It is interesting to note that the upper bound assumed in 1.3 implies the asymptotic formula 1.4 .

2. We may assume a version of (1.3) with a main term of type $x P_{d}(\log x, k)$, where $P_{d}(X, k)$ is a polynomial of degree $d$, and get analogous consequences for $F \otimes \bar{F}(s)$.

3. In general, $\sigma_{a}(F \otimes \bar{F})=1$ does not imply that $\sigma_{a}(F)=1$. Choose indeed $a(n)=n^{1 / 4}$ if $n$ is a square and $a(n)=0$ otherwise; in this case it is easily seen that $\sigma_{a}(F \otimes \bar{F})=1$ but $\sigma_{a}(F)=5 / 8$; such coefficients do not satisfy the Ramanujan condition. 
4. It is well known that the function $L(s, f \otimes \bar{f})$ introduced above may have, in addition to the pole at $s=1$, also poles on the line $\sigma=1 / 4$; see again Chapter 13 of [2]. Therefore we cannot expect $(s-1) F \otimes \bar{F}(s)$ to have a holomorphic continuation to a much wider right half-plane than $\sigma>\theta$.

5. A final remark on the above corollary, namely it is not true that $\sigma_{a}(F)=1$ for every $F \in \mathcal{S}^{\sharp}$. Indeed, it follows from Theorem 1 of [7] that for every $F \in \mathcal{S}^{\sharp}$ we have $\sigma_{a}(F) \geq\left(d_{F}+1\right) / 2 d_{F}$, and in [10] (see p. 1347) we have shown that there exist $F \in \mathcal{S}^{\sharp}$ with $\sigma_{a}(F)$ arbitrarily close to $1 / 2$; our examples in [10] do not satisfy the Ramanujan condition.

Acknowledgments. This research was partially supported by PRIN-2015 Number Theory and Arithmetic Geometry and by the Polish National Science Centre grant number 2017/25/B/ST1/00208. A.P. is a member of GNAMPA.

\section{Proofs}

2.1. Lemmas. We first obtain a suitable version of the basic transformation formula for the linear twists $F(s, \alpha)$ of functions $F \in \mathcal{S}^{\sharp}$ of degree 2, defined for $\alpha>0$ and $\sigma>1$ by

$$
F(s, \alpha)=\sum_{n=1}^{\infty} \frac{a(n) e(-n \alpha)}{n^{s}},
$$

obtained in Theorem 1.2 of [9]. We recall that $\theta_{F}$ is the internal shift defined in Section 1. Lemma 1. Let $F \in \mathcal{S}^{\sharp}$ with degree 2 and conductor $q$, and let $\alpha>0$. Then there exists a polynomial $Q(s)$ such that

$$
\begin{aligned}
F(s, \alpha)= & \frac{Q(s)}{\alpha} F(s+1, \alpha)+(1+q \alpha)^{2 s-1+2 i \theta_{F}} \\
& \times\left\{F\left(s, \frac{\alpha}{1+q \alpha}\right)-\frac{q \alpha+1}{\alpha} Q(s) F\left(s+1, \frac{\alpha}{1+q \alpha}\right)\right\}+H(s, \alpha),
\end{aligned}
$$

where $H(s, \alpha)$ is holomorphic for $\sigma>0$ and differentiable with continuous derivative for $\alpha>0$.

Proof. We start with the transformation formula in Theorem 1.2 of [9] with the choice $K=1$, which we rewrite as

$$
\begin{array}{r}
F(s, \alpha)=c_{1} q^{s} \alpha^{2 s-1+2 i \theta_{F}}\left\{\bar{F}\left(s+2 i \theta_{F},-\frac{1}{q \alpha}\right)+c_{2} \alpha Q_{1}(s) \bar{F}\left(s+1+2 i \theta_{F},-\frac{1}{q \alpha}\right)\right\} \\
+H_{1}(s, \alpha) .
\end{array}
$$

Here $Q_{1}(s)$ is a certain polynomial, $c_{j} \neq 0, j=1,2$, are constants depending on $F(s)$, and $H_{1}(s, \alpha)$ is holomorphic for $\sigma>0$ and continuously differentiable for $\alpha>0$. The fact that $H_{1}(s, \alpha)$ is continuously differentiable for $\alpha>0$, which will be important later on in the paper, follows easily from the proof of the above mentioned Theorem 1.2, since $\frac{\partial}{\partial \alpha} H_{1}(s, \alpha)$ can be expressed, for $\sigma>0$ and $\alpha>0$, in terms of absolutely and uniformly convergent series of continuous functions in $\alpha$. Note that formally Theorem 1.2 in [9] asserts that $H_{1}(s, \alpha)$ is holomorphic in a smaller region, but actually the condition 
on the region is needed only for the bound on $H_{1}(s, \alpha)$ stated immediately after. Since $F(s, \alpha)$ is 1-periodic in $\alpha, 2.2$ yields

$$
\begin{aligned}
& \alpha^{2 s-1+2 i \theta_{F}}\left\{\bar{F}\left(s+2 i \theta_{F},-\frac{1}{q \alpha}\right)+c_{2} \alpha Q_{1}(s) \bar{F}\left(s+1+2 i \theta_{F},-\frac{1}{q \alpha}\right)\right\} \\
& =(\alpha+1)^{2 s-1+2 i \theta_{F}} \\
& \quad \times\left\{\bar{F}\left(s+2 i \theta_{F},-\frac{1}{q(\alpha+1)}\right)+c_{2}(\alpha+1) Q_{1}(s) \bar{F}\left(s+1+2 i \theta_{F},-\frac{1}{q(\alpha+1)}\right)\right\} \\
& \quad+H_{2}(s, \alpha),
\end{aligned}
$$

where $H_{2}(s, \alpha)$ has the same properties as $H_{1}(s, \alpha)$. Lemma 1 follows now changing first $s+2 i \theta_{F} \mapsto s$, then taking conjugates on both sides and finally changing $\alpha \mapsto 1 /(q \alpha)$ and $s \mapsto \bar{s}$.

For later reference, we note that changing $s \mapsto \bar{s}$ and taking conjugates in 2.1 we obtain also

$$
\begin{aligned}
\bar{F}(s,-\alpha)= & \frac{\bar{Q}(s)}{\alpha} \bar{F}(s+1,-\alpha)+(1+q \alpha)^{2 s-1-2 i \theta_{F}} \\
& \times\left\{\bar{F}\left(s,-\frac{\alpha}{1+q \alpha}\right)-\frac{q \alpha+1}{\alpha} \bar{Q}(s) \bar{F}\left(s+1,-\frac{\alpha}{1+q \alpha}\right)\right\}+\bar{H}(s, \alpha),
\end{aligned}
$$

where $\bar{H}(s, \alpha)$ has the same properties of $H(s, \alpha)$ and $\bar{Q}(s)$ is a polynomial.

Given a test function $\phi \in C_{0}^{\infty}((0, \infty))$ and $F \in \mathcal{S}^{\sharp}$ we define

$$
h_{\phi}(s)=\int_{0}^{\infty} \phi(\alpha)\left(1-(1+q \alpha)^{2(s-1)}\right) \mathrm{d} \alpha,
$$

where $q>0$ is the conductor of $F(s)$. Clearly, $h_{\phi}(s)$ is an entire function. The link between the shifted convolutions and the Rankin-Selberg convolution is provided by the following basic lemma.

Lemma 2. Let $F \in \mathcal{S}^{\sharp}$ with $d=2, F_{k}(s)$ be as in 1.2 and $\sigma>\sigma_{a}(F \otimes \bar{F})$. Then for any test function $\phi \in C_{0}^{\infty}((0, \infty))$ we have

$$
h_{\phi}(s) F \otimes \bar{F}(s)=\sum_{k=1}^{\infty} A_{k}(s) F_{k}(s)+\sum_{k=1}^{\infty} B_{k}(s) \bar{F}_{k}(s)+H(s),
$$

where $H(s)$ is holomorphic for $\sigma>\max \left(1 / 2, \sigma_{a}(F \otimes \bar{F})-1\right)$ and $A_{k}(s), B_{k}(s)$ are entire and bounded by $O_{C}\left(k^{-C}\right)$ for every $C>0$, uniformly for $s$ in any compact subset of $\mathbb{C}$.

Proof. For $\sigma>1$ we consider the integral

$$
I(s)=\int_{0}^{\infty} \phi(\alpha) F(s, \alpha) \bar{F}(s,-\alpha) \mathrm{d} \alpha,
$$


and we compute $I(s)$ in two different ways. First, a term-by-term integration shows that

$$
\begin{aligned}
I(s) & =\sum_{n=1}^{\infty} \sum_{m=1}^{\infty} \frac{a(n) \overline{a(m)}}{n^{s} m^{s}} \int_{0}^{\infty} \phi(\alpha) e(-(n-m) \alpha) \mathrm{d} \alpha=\sum_{n=1}^{\infty} \sum_{m=1}^{\infty} \frac{a(n) \overline{a(m)}}{n^{s} m^{s}} \widehat{\phi}(n-m) \\
& =\widehat{\phi}(0) F \otimes \bar{F}(2 s)+\sum_{k=1}^{\infty} \sum_{n=1}^{\infty} \frac{a(n) \overline{a(n+k)}}{n^{s}(n+k)^{s}} \widehat{\phi}(-k)+\sum_{k=1}^{\infty} \sum_{n=1}^{\infty} \frac{\overline{a(n)} a(n+k)}{n^{s}(n+k)^{s}} \widehat{\phi}(k) \\
& =\widehat{\phi}(0) F \otimes \bar{F}(2 s)+\Sigma(s)+\bar{\Sigma}(s),
\end{aligned}
$$

say. Moreover, since $\phi(\alpha)$ is a smooth test function, for $k \in \mathbb{Z}, k \neq 0$, and any $C>0$ we have

$$
\widehat{\phi}(k) \ll_{C}|k|^{-C} \text {. }
$$

Thanks to the sharp decay of $\widehat{\phi}(k)$ in $(2.8)$, for any $\varepsilon, C>0$ we have

$$
\begin{aligned}
\Sigma(s)= & \sum_{n=1}^{\infty} \frac{a(n)}{n^{s}} \sum_{1 \leq k \leq n^{\varepsilon}} \widehat{\phi}(-k) \frac{\overline{a(n+k)}}{(n+k)^{s}}+O\left(\sum_{n=1}^{\infty} \frac{|a(n)|}{n^{\sigma}} \sum_{k>n^{\varepsilon}} \frac{|a(n+k)|}{|n+k|^{\sigma} k^{C / \varepsilon}}\right) \\
= & \sum_{n=1}^{\infty} \frac{a(n)}{n^{2 s}} \sum_{1 \leq k \leq n^{\varepsilon}} \widehat{\phi}(-k) \overline{a(n+k)} \\
& +\sum_{n=1}^{\infty} \frac{a(n)}{n^{s}} \sum_{1 \leq k \leq n^{\varepsilon}} \widehat{\phi}(-k) \overline{a(n+k)}\left(\frac{1}{(n+k)^{s}}-\frac{1}{n^{s}}\right)+f_{1}(s),
\end{aligned}
$$

say, where $f_{1}(s)$ is an entire function. We denote by $f_{2}(s)$ the second term in the last equation in 2.9; ; again thanks to 2.8, and to Cauchy-Schwarz inequality, we have

$$
\begin{aligned}
f_{2}(s) & \ll|s| \sum_{n=1}^{\infty} \frac{|a(n)|}{n^{\sigma}} \sum_{1 \leq k \leq n^{\varepsilon}} k|\widehat{\phi}(-k)| \frac{|a(n+k)|}{n^{\sigma+1}} \\
& \ll|s| \sum_{k=1}^{\infty} k|\widehat{\phi}(-k)| \sum_{n \geq k^{1 / \varepsilon}} \frac{|a(n) a(n+k)|}{n^{2 \sigma+1}} \ll|s| \sum_{n=1}^{\infty} \frac{|a(n)|^{2}}{n^{2 \sigma+1}},
\end{aligned}
$$

hence $f_{2}(s)$ is holomorphic for $2 \sigma>\sigma_{a}(F \otimes \bar{F})-1$. The first term in the last equation in (2.9) equals

$$
\begin{aligned}
\sum_{k=1}^{\infty} \widehat{\phi}(-k) \sum_{n \geq k^{1 / \varepsilon}} \frac{a(n) a(n+k)}{n^{2 s}}= & \sum_{k=1}^{\infty} \widehat{\phi}(-k) F_{k}(2 s) \\
& +O\left(\sum_{k=1}^{\infty}|\widehat{\phi}(-k)| \sum_{n<k^{1 / \varepsilon}} \frac{|a(n) a(n+k)|}{n^{2 \sigma}}\right) \\
= & \sum_{k=1}^{\infty} \widehat{\phi}(-k) F_{k}(2 s)+O\left(\sum_{k=1}^{\infty}|\widehat{\phi}(-k)| k^{c}\right) \\
& =\sum_{k=1}^{\infty} \widehat{\phi}(-k) F_{k}(2 s)+f_{3}(s),
\end{aligned}
$$

say, where $c=c(\sigma, \varepsilon)$ is a certain constant. Hence, once more thanks to $(2.8), f_{3}(s)$ is an 
entire function. Therefore, collecting the above results we have

$$
\Sigma(s)=\sum_{k=1}^{\infty} \widehat{\phi}(-k) F_{k}(2 s)+f_{4}(s)
$$

where $f_{4}(s)$ is holomorphic for $2 \sigma>\sigma_{a}(F \otimes \bar{F})-1$. In a completely analogous way we also obtain

$$
\bar{\Sigma}(s)=\sum_{k=1}^{\infty} \widehat{\phi}(k) \bar{F}_{k}(2 s)+f_{5}(s)
$$

again $f_{5}(s)$ being holomorphic for $2 \sigma>\sigma_{a}(F \otimes \bar{F})-1$. From (2.7), 2.10) and 2.11) we finally obtain

$$
I(s)=\widehat{\phi}(0) F \otimes \bar{F}(2 s)+\sum_{k=1}^{\infty} \widehat{\phi}(-k) F_{k}(2 s)+\sum_{k=1}^{\infty} \widehat{\phi}(k) \bar{F}_{k}(2 s)+f(s),
$$

where $f(s)$ is holomorphic for $2 \sigma>\sigma_{a}(F \otimes \bar{F})-1$.

Next we compute the integral in 2.6 using the transformation formulae 2.1 and (2.3). With obvious notation, for $\sigma>1$ we rewrite $(2.1)$ and $(2.3)$ as

$$
F(s, \alpha)=\sum_{j=1}^{4} G_{j}(s, \alpha) \quad \text { and } \quad \bar{F}(s,-\alpha)=\sum_{\ell=1}^{4} K_{\ell}(s, \alpha),
$$

hence plugging 2.13 into 2.6 we obtain

$$
I(s)=\sum_{j=1}^{4} \sum_{\ell=1}^{4} \int_{0}^{\infty} \phi(\alpha) G_{j}(s, \alpha) K_{\ell}(s, \alpha) \mathrm{d} \alpha=\sum_{j=1}^{4} \sum_{\ell=1}^{4} I_{j, \ell}(s),
$$

say. Clearly,

$$
I_{j, \ell}(s) \text { is holomorphic for } \sigma>0 \text { for every } j, \ell \in\{1,3,4\} \text {. }
$$

Switching summation and integration we have

$$
I_{1,2}(s)=Q(s) \sum_{n=1}^{\infty} \sum_{m=1}^{\infty} \frac{a(n) \overline{a(m)}}{n^{s+1} m^{s}} \int_{0}^{\infty} \frac{\phi(\alpha)}{\alpha}(1+q \alpha)^{2 s-1-2 i \theta_{F}} e\left(-n \alpha+m \frac{\alpha}{1+q \alpha}\right) \mathrm{d} \alpha,
$$

and, writing $\alpha^{-1}(1+q \alpha)^{2 s-1-2 i \theta_{F}}=\rho_{1}(\alpha, s)+i \rho_{2}(\alpha, s)$ with real functions $\rho_{j}(\alpha, s)$, $j=1,2$, we express the above integral as $I_{1}(s, n, m)+i I_{2}(s, n, m)$ with

$$
\int_{0}^{\infty} \phi(\alpha) \rho_{j}(\alpha, s) e\left(-n \alpha+m \frac{\alpha}{1+q \alpha}\right) \mathrm{d} \alpha .
$$

Since $\rho_{j}(\alpha, s)$ is clearly continuously differentiable in $\alpha$, the total variation $V_{j}(s)$ of $\phi(\alpha) \rho_{j}(\alpha, s)$ for $\alpha \in(0, \infty)$ is uniformly bounded for $s$ in any compact subset of $\mathbb{C}$. Let

$$
f_{n, m}(\alpha)=-n \alpha+m \frac{\alpha}{1+q \alpha} \quad \text { and } \quad V(s)=\max \left(V_{1}(s), V_{2}(s)\right) .
$$

It is easily seen, by checking its second derivative, that $f_{n, m}(\alpha)$ has a monotonic first derivative for $\alpha>0$ for every $n, m \geq 1$. Therefore, for $n, m \geq 1$ we may apply the first and second derivative tests to the integrals $I_{j}(s, n, m)$ in $(2.16)$, see Lemmas 5.1.2 
and 5.1.3 of Huxley [1]. Since

$$
f_{n, m}^{\prime}(\alpha)=-n+\frac{m}{(1+q \alpha)^{2}} \quad \text { and } \quad f_{n, m}^{\prime \prime}(\alpha)=-\frac{2 m q}{(1+q \alpha)^{3}},
$$

for $j=1,2$ we obtain for suitable constants $0<a<b$ :

$$
I_{j}(s, n, m) \ll \begin{cases}V(s) / n & \text { if } 1 \leq m \leq a n \\ V(s) / \sqrt{m} & \text { if } \text { an }<m<b n \\ V(s) / m & \text { if } m \geq b n,\end{cases}
$$

where $a, b$ and the implied constant in the «-symbol may depend on $\phi(\alpha)$ and $F(s)$. As a consequence, for $j=1,2$ we have

$$
\begin{aligned}
& \sum_{n=1}^{\infty} \sum_{m=1}^{\infty}\left|\frac{a(n) \overline{a(m)}}{n^{s+1} m^{s}} I_{j}(s, n, m)\right| \\
& \ll \sum_{n=1}^{\infty} \frac{|a(n)|}{n^{\sigma+2}} \sum_{m \leq a n} \frac{|a(m)|}{m^{\sigma}}+\sum_{n=1}^{\infty} \frac{|a(n)|}{n^{\sigma+1}} \sum_{a n<m<b n} \frac{|a(m)|}{m^{\sigma+1 / 2}}+\sum_{n=1}^{\infty} \frac{|a(n)|}{n^{\sigma+1}} \sum_{m \geq b n} \frac{|a(m)|}{m^{\sigma+1}} \\
& \ll \sum_{n=1}^{\infty} \frac{|a(n)|}{n^{2 \sigma+1-\varepsilon}}+\sum_{n=1}^{\infty} \frac{|a(n)|}{n^{2 \sigma+1 / 2-\varepsilon}}+\sum_{n=1}^{\infty} \sum_{m=1}^{\infty} \frac{|a(n) a(m)|}{n^{\sigma+1} m^{\sigma+1}},
\end{aligned}
$$

and all the sums in the last row are uniformly convergent in any compact subset of the half-plane $2 \sigma>1 / 2$. Hence

$$
I_{1,2}(s) \text { is holomorphic for } 2 \sigma>1 / 2,
$$

and similar arguments show that

$$
I_{2,1}(s), I_{2,3}(s) \text { and } I_{3,2}(s) \text { are holomorphic for } 2 \sigma>1 / 2 .
$$

The integral $I_{2,4}(s)$ can be treated using only the first derivative test. Switching summation and integration we have

$$
I_{2,4}(s)=\sum_{n=1}^{\infty} \frac{a(n)}{n^{s}} \int_{0}^{\infty} \phi(\alpha)(1+q \alpha)^{2 s-1+2 i \theta_{F}} \bar{H}(s, \alpha) e\left(-n \frac{\alpha}{1+q \alpha}\right) \mathrm{d} \alpha
$$

and, again splitting $(1+q \alpha)^{2 s-1+2 i \theta_{F}} \bar{H}(s, \alpha)$ into real and imaginary parts, we rewrite the above integral as $I_{1}(s, n)+i I_{2}(s, n)$ with

$$
I_{j}(s, n)=\int_{0}^{\infty} \phi(\alpha) \rho_{j}(\alpha, s) e\left(-n \frac{\alpha}{1+q \alpha}\right) \mathrm{d} \alpha
$$

and certain real functions $\rho_{j}(\alpha, s)$. Here the $\rho_{j}(\alpha, s)$ 's have the same properties as in (2.16), thanks to the corresponding properties of $\bar{H}(s, \alpha)$ in 2.3 . Moreover, by the first derivative test we have

$$
I_{j}(s, n) \ll \frac{V(s)}{n},
$$

where $V(s)$ is the maximum of the total variations of $\phi(\alpha) \rho_{j}(\alpha, s), j=1,2$, for $\alpha \in(0, \infty)$. As a consequence we have

$$
I_{2,4}(s) \ll \sum_{n=1}^{\infty} \frac{|a(n)|}{n^{\sigma+1}}
$$


Since a completely similar argument can be applied to $I_{4,2}(s)$, we deduce that

$$
I_{2,4}(s) \text { and } I_{4,2}(s) \text { are holomorphic for } \sigma>0 .
$$

Finally we deal with $I_{2,2}(s)$. Here we have

$$
\begin{aligned}
I_{2,2}(s) & =\sum_{n=1}^{\infty} \sum_{m=1}^{\infty} \frac{a(n) \overline{a(m)}}{n^{s} m^{s}} \int_{0}^{\infty} \phi(\alpha)(1+q \alpha)^{2(2 s-1)} e\left(-(n-m) \frac{\alpha}{1+q \alpha}\right) \mathrm{d} \alpha \\
& =F \otimes \bar{F}(2 s) \int_{0}^{\infty} \phi(\alpha)(1+q \alpha)^{2(2 s-1)} \mathrm{d} \alpha+J_{2,2}(s)+\bar{J}_{2,2}(s),
\end{aligned}
$$

where

$$
J_{2,2}(s)=\sum_{k=1}^{\infty} \sum_{n=1}^{\infty} \frac{a(n)}{n^{s}} \frac{\overline{a(n+k)}}{(n+k)^{s}} \int_{0}^{\infty} \phi(\alpha)(1+q \alpha)^{2(2 s-1)} e\left(k \frac{\alpha}{1+q \alpha}\right) \mathrm{d} \alpha
$$

and $\bar{J}_{2,2}(s)=\overline{J_{2,2}(\bar{s})}$. After the substitution $\frac{\alpha}{1+q \alpha} \mapsto \alpha$ the integral in 2.21 becomes the Fourier transform at $-k$ of a certain smooth function with compact support in $(0, \infty)$ which we denote by $\phi(\alpha, s)$. By the same argument leading to 2.10 and 2.11 we therefore obtain

$$
J_{2,2}(s)=\sum_{k=1}^{\infty} \widehat{\phi}(-k, s) F_{k}(2 s)+g_{1}(s) \quad \text { and } \quad \bar{J}_{2,2}(s)=\sum_{k=1}^{\infty} \widehat{\phi}(k, s) \bar{F}_{k}(2 s)+g_{2}(s),
$$

where $\widehat{\phi}(k, s)$ is entire and bounded by $O_{C}\left(k^{-C}\right)$ for every $C>0$, uniformly for $s$ in any compact subset of $\mathbb{C}$, and $g_{1}(s), g_{2}(s)$ are holomorphic for $2 \sigma>\sigma_{a}(F \otimes \bar{F})-1$. Hence 2.20 and 2.22 show that

$$
\begin{aligned}
I_{2,2}(s)= & F \otimes \bar{F}(2 s) \int_{0}^{\infty} \phi(\alpha)(1+q \alpha)^{2(2 s-1)} \mathrm{d} \alpha \\
& +\sum_{k=1}^{\infty} \widehat{\phi_{1}}(-k, s) F_{k}(2 s)+\sum_{k=1}^{\infty} \widehat{\phi_{1}}(k, s) \bar{F}_{k}(2 s)+g_{3}(s)
\end{aligned}
$$

with $g_{3}(s)$ holomorphic for $2 \sigma>\sigma_{a}(F \otimes \bar{F})-1$.

From 2.14), 2.15, 2.17), 2.18, 2.19 and 2.23 we finally get

$$
\begin{aligned}
I(s)= & F \otimes \bar{F}(2 s) \int_{0}^{\infty} \phi(\alpha)(1+q \alpha)^{2(2 s-1)} \mathrm{d} \alpha \\
& +\sum_{k=1}^{\infty} \widehat{\phi_{1}}(-k, s) F_{k}(2 s)+\sum_{k=1}^{\infty} \widehat{\phi_{1}}(k, s) \bar{F}_{k}(2 s)+g(s),
\end{aligned}
$$

where $g(s)$ is holomorphic for $2 \sigma>\max \left(1 / 2, \sigma_{a}(F \otimes \bar{F})-1\right)$. In view of 2.8 and of the properties of $\widehat{\phi}(k, s)$ reported after 2.22), Lemma 2 now follows by comparing 2.12) with (2.24) and changing $2 s \mapsto s$.

The next lemma contains the properties of the function $h_{\phi}(s)$, defined in $(2.4)$, required in the proof of our theorem. Note that such properties do not depend on the value of the conductor $q$.

LEMmA 3. Let $\phi \in C_{0}^{\infty}((0, \infty)), \phi(x) \geq 0$ but not identically vanishing. Then the entire function $h_{\phi}(s)$ has a simple zero at $s=1$ and $h_{\phi}(s) \neq 0$ for $s \in \mathbb{R} \backslash\{1\}$. 
Proof. Clearly we have $h_{\phi}(1)=0$ and

$$
-h_{\phi}^{\prime}(1)=2 \int_{0}^{\infty} \phi(\alpha) \log (1+q \alpha) \mathrm{d} \alpha>0,
$$

hence $h_{\phi}(s)$ has a simple zero at $s=1$. Moreover, it is clear from 2.4 that $h_{\phi}(s)>0$ for $s<1$, and $h_{\phi}(s)<0$ for $s>1$.

2.2. Proof of the theorem and its corollary. Let $\varepsilon>0$ be as in 1.3 . Since clearly $\sigma_{a}(F \otimes \bar{F}) \leq 2$, by partial summation, 1.1 and 1.3 we have

$$
\begin{aligned}
\sum_{n=1}^{\infty} \frac{a(n) \overline{a(n+k)}}{n^{s}} & =s \int_{1}^{\infty} S_{k}(x) x^{-s-1} \mathrm{~d} x \\
& \ll|s| \int_{1}^{k^{1 / \varepsilon}} x^{1+\varepsilon}(x+k)^{1+\varepsilon} x^{-\sigma-1} \mathrm{~d} x+|s| \int_{k^{1 / \varepsilon}}^{\infty} x^{\theta-\sigma-1} \mathrm{~d} x \ll|s| k^{2 / \varepsilon}
\end{aligned}
$$

provided $\sigma \geq \theta+\delta$, for any $\delta>0$. Hence $F_{k}(s)$ is holomorphic for $\sigma>\theta$ and satisfies $F_{k}(s) \ll k^{2 / \varepsilon}$ uniformly for $s$ in any compact subset of the half-plane $\sigma>\theta$; the same holds for $\bar{F}_{k}(s)$. Applying Lemma 2 with $C=2 / \varepsilon+2$ and recalling that $\theta>1 / 2$, we deduce that the right hand side of $(2.5)$ is holomorphic for $\sigma>\max \left(\theta, \sigma_{a}(F \otimes \bar{F})-1\right)$. Hence from Lemma 3 we see that $F \otimes \vec{F}(s)$ is meromorphic in the same half-plane, and its only real singularity in such half-plane is at most a simple pole at $s=1$. Since by the hypothesis of the theorem we infer that $\max \left(\theta, \sigma_{a}(F \otimes \bar{F})-1\right)<\sigma_{a}(F \otimes \bar{F})$, from Landau's theorem on Dirichlet series with non-negative coefficients we deduce that $F \otimes \bar{F}(s)$ has a simple pole at $s=1$ and $\sigma_{a}(F \otimes \bar{F})=1$; the theorem is therefore proved.

Moving on to the corollary, the asymptotic formula (1.4) follows from the theorem by a standard Tauberian theorem, since the coefficients of $F \otimes \bar{F}(s)$ are non-negative. Moreover, under the Ramanujan condition, by the Cauchy-Schwarz inequality and (1.4) we have

$$
\sum_{n \leq x}|a(n)| \ll x \quad \text { and } \quad x \ll \sum_{n \leq x}|a(n)|^{2} \ll x^{\varepsilon} \sum_{n \leq x}|a(n)|
$$

for every $\varepsilon>0$, hence $\sigma_{a}(F)=1$.

\section{References}

[1] M. N. Huxley, Area, Lattice Points and Exponential Sums, London Math. Soc. Monogr. (N.S.) 13, Oxford Science Publ., Clarendon Press, New York, 1996.

[2] H. Iwaniec, Topics in Classical Automorphic Forms, Grad. Stud. Math. 17, Amer. Math. Soc., Providence, 1997.

[3] M. Jutila, The additive divisor problem and its analogs for Fourier coefficients of cusp forms. I, Math. Z. 223 (1996), 435-461.

[4] J. Kaczorowski, Axiomatic theory of L-functions: the Selberg class, in: Analytic Number Theory (Cetraro, 2002), Lecture Notes in Math. 1891, Springer, Berlin, 2006, 133-209.

[5] J. Kaczorowski, A. Perelli, The Selberg class: a survey, in: Number Theory in Progress, de Gruyter, Berlin, 1999, 953-992.

[6] J. Kaczorowski, A. Perelli, On the structure of the Selberg class, V: $1<d<5 / 3$, Invent. Math. 150 (2002), 485-516. 
[7] J. Kaczorowski, A. Perelli, On the structure of the Selberg class, VI: Non-linear twists, Acta Arith. 116 (2005), 315-341.

[8] J. Kaczorowski, A. Perelli, On the structure of the Selberg class, VII: $1<d<2$, Ann. of Math. (2) 173 (2011), 1397-1441.

[9] J. Kaczorowski, A. Perelli, Twists, Euler products and a converse theorem for L-functions of degree 2, Ann. Sc. Norm. Super. Pisa Cl. Sci. (5) 14 (2015), 441-480.

[10] J. Kaczorowski, A. Perelli, Some remarks on the convergence of the Dirichlet series of L-functions and related questions, Math. Z. 285 (2017), 1345-1355.

[11] A. Perelli, A survey of the Selberg class of L-functions, part I, Milan J. Math. 73 (2005), 19-52.

[12] A. Perelli, A survey of the Selberg class of L-functions, part II, Riv. Mat. Univ. Parma (7) $3^{*}$ (2004), 83-118.

[13] A. Perelli, Non-linear twists of L-functions: a survey, Milan J. Math. 78 (2010), 117-134.

[14] A. Perelli, Converse theorems: from the Riemann zeta function to the Selberg class, Boll. Unione Mat. Ital. 10 (2017), 29-53. 
